\title{
Case Study of a Clean Energy Solution by Employing the Distributed Energy Sources Based on Perovskite Solar Cells
}

\author{
Zhihao Li ${ }^{1}$, Kuan Way Chee ${ }^{2,3}$, Zhenhai Yang ${ }^{2,3}$, Jiapeng Su${ }^{1}$, Anjun Jin ${ }^{1, *}$ \\ ${ }^{1}$ Laboratory of Renewable Energy Research, Faculty of Science and Engineering, Ningbo University, Ningbo, China \\ ${ }^{2}$ Department of Electrical and Electronic Engineering, Faculty of Science and Engineering, University of Nottingham, Ningbo, China \\ ${ }^{3}$ Key Laboratory of Marine Materials and Related Technologies, Zhejiang Key Laboratory of Marine Materials and Protective Technologies, \\ Ningbo Institute of Materials Technology and Engineering, Chinese Academy of Sciences, Ningbo, China
}

\author{
Email address: \\ ajjin@nbu.edu.cn (Anjun Jin) \\ ${ }^{*}$ Corresponding author
}

\section{To cite this article:}

Zhihao Li, Kuan Way Chee, Zhenhai Yang, Jiapeng Su, Anjun Jin. Case Study of a Clean Energy Solution by Employing the Distributed Energy Sources Based on Perovskite Solar Cells. American Journal of Science, Engineering and Technology. Special Issue: Advances in Thermoelectric Generation and Renewable Energies. Vol. 5, No. 2, 2020, pp. 96-104. doi: 10.11648/j.ajset.20200502.17

Received: MM DD, 2020; Accepted: MM DD, 2020; Published: June 18, 2020

\begin{abstract}
This article presents study results of an emerging technology from the distributed energy sources (DES). In order that a clean energy technology becomes a main-stream player of the DES, it is imperative that this technology achieves well in several aspects of performance in terms of a high efficiency, good stability, and long life time. As the perovskite solar cells (PSC) has rapidly improved in all these aspects to produce solar energy, as a result, the solution from PSC is to be advantageous in performance and, moreover, that can offer low cost. In comparison to older methods in solar cells than PSC, the PSC is highly favored for their wide, tunable bandgap, and solution processability. Currently the single-junction PSC offer comparable or better efficiencies to those in the mainstream renewable energy methods [of the single- crystalline silicon, cadmium telluride, and copper indium gallium selenide]. Nevertheless, the energy storage is indispensable for the power stability solution in application to the localized energy consumption [for the main power provider]. Furthermore, the authors have studied several methods to design the best-distributed energy system that implements the alternative energies, energy storage, and the advanced materials for the alternative energy generation. Finally, authors will present an integrated system that enables a maximum energy output and that includes key factors as the alternative energy generation, energy storage, and a distributed energy system.
\end{abstract}

Keywords: Distributed Energy Source, Renewable Energy, Perovskite Solar Cell, Energy Storage, Energy Management System, High Efficiency

\section{Introduction}

Authors employ this article to investigate and review several insightful indicators related to the current alternative energies $[\mathrm{AE}] ; \mathrm{AE}$ technologies and their application have been of tremendous interest and of great importance in reducing the climate changes referring to Clark et al. [1, 2]. The alternative energies have been established that is recorded firstly in the 1978s in the United States [3].

In response to the pressing need in the carbon economy and climate changes in recent years, $\mathrm{AE}$ generation and strategic commercial interests lead to significant advances over the past decades in clean technologies [1], also known as Clean Technology. Key resources of alternative energy generation include the following: solar, wind, hydro, tidal, and geothermal biomass, hydrogen fuel cell, thermal energy conversion, nuclear energy, and ocean energy conversion technologies, etc.

The great endeavor in research and development has been dedicated to improving the output of the distributed energies. In order to adopt these technologies, it is imperative that the power output has good stability, high efficiency, and superior 
energy management. This article is dedicated to these technologies.

Moreover, there are huge endeavors dedicated to wisely study the alternative energies over prior two decades; with noteworthy commercial application on a large scale. There are interesting technologies and exciting ones that have recently emerged. It is hopeful that the variable power generation, with the advent of plentiful energy storage, can be sufficiently utilized more than it has been so far [4].

As an example, authors will study both solar power and energy storage from the supply side in this article. Solar photovoltaic (PV) cells are promising for clean energy sources as demonstrated in the NREL database [5]. The solar energy may have significant power output and efficiency, but it must be managed with the load-leveling so that its output is suited to the demand profiles. The energy output, a modeling database (library), and data analysis can be consequently fed into a smart grid. The smart grid may respond to demand request employing various conditions including energy storage, sources, and electrical reliability [1].

The top-three prevailing types of solar cells for the commercialized solar cells are based on polysilicon, single crystalline silicon, and amorphous silicon (thin film). The impacts of the first-generation PVs are as follows:

1) Polysilicon is the most popular; its efficiency ranges from $13 \%$ and up in the field to $18 \%$ in the lab;

2) Single crystalline silicon solar cells have higher efficiency, that is from $14 \%$ and up in the field to $24 \%$ in the lab;

3) Amorphous silicon (thin film) is cheap; the efficiency is low. That is from $5 \%$ and up in the field to $13 \%$ in the lab.

The current state of the perovskite solar cells demonstrates as a rising star in solar cell applications which can be a potential technology disruptor. Researchers have discovered that the material advance in the perovskite solar cells are extremely exciting [5]. This advance can lower the cost in the solar power generation and has a promise future to accelerate the achievement of the grid parity for the energy distribution when the cost to utilize the clean technology is the same as the electric bill to use the grid power that is traditionally powered by the fossil-based fuel (s). The authors believe that the materials advancement in the near future can achieve the significant stability, the manufacturing capability, and lower cost than the present solar PV to meet the expectation on the solar renewable energy. The single-junction efficiencies of these perovskite solar cells are comparable to those of multicrystalline silicon, cadmium telluride and copper indium gallium selenide. In this article, the authors will focus on their studies as follows: efficiency is high, stability is being addressed, and the manufacturing cost is projected to be easier thus the cost to be much lower.

The advancement of PSC is emerging as a promising leader of the next generation photovoltaic technology; that has achieved very high efficiency [6-10] and rapidly improved its stabilities in some case that, for example, has organic-inorganic perovskite interfaces [8, 11-12].
PSC advantages also include their widely tunable bandgaps [of the material]. The optimized bandgap of the perovskite-perovskite tandem photovoltaics has benefited the advancement of the efficient PSC in tandem structure [13] and interface engineering techniques [11, 14].

One of the limitations for solar energy is that sometimes the sun is not shining enough or that there is no sunlight. To tackle the intermittent issues or unstable generation of the solar PV power generation, the solar PV power can be stored through one of the energy storage solutions.

In order to accomplish the smooth power output, a number of the energy storage technologies are available. Among various energy storage solutions, the Li-ion battery [15] is recently becoming one of the most attractive solutions [1619]. Since Yoshio [15] created the first commercially viable lithium ion battery, the Li-ion battery has been greatly improved in recent years and extensively commercialized. The battery has reasonable cost and reasonably long life today, for example, the life time of a Li-ion battery can last from three years or 1200-cycles whichever comes sooner.

Finally, anything that can achieve an efficiency above or at a level of $26 \%$ that is the best efficiency of single crystal silicon solar cells [6-10] with sufficiently long life [8].

It may be very appealing success in the field of solar energy technology. [20-21]

\section{Methods and Results}

\subsection{Perovskite Solar Cell Efficiency}

The application of perovskite materials in photovoltaics can be traced back to the first discovery of photocurrent in $\mathrm{BaTiO}_{3}$ in 1956 [22]. Subsequently, the photovoltaic effect in $\mathrm{LiNbO}_{3}$ and other materials was also reported, which was mainly ascribed to the electric field formed on the surface of crystals [23]. However, the efficiency reported in those early studies was very low, usually below 1\%. By 2012, photoelectric conversion efficiencies of $9.7 \%$ and $10.9 \%$ were achieved in perovskite solar cells based on $\mathrm{CH}_{3} \mathrm{NH}_{3} \mathrm{PbI}_{3}$ and $\mathrm{CH}_{3} \mathrm{NH}_{3} \mathrm{PbI}_{3}-\mathrm{XClX}$ [24-25], respectively, opening a new chapter in this type of third generation PV. In early 2013, a further enhancement in efficiency exceeding $12.3 \%$ was attained in low-temperature processed perovskite-based meso-superstructured solar cells [24-26]. Later that year, a sequential deposition method was reported for the fabrication of perovskite-sensitized mesoscopic solar cells, which enabled excellent photovoltaic performance up to a higher efficiency of $15 \%$ [27]. In the same year, the efficiency was further improved to $15.4 \%$ by incorporating vapor-deposited perovskite as the absorbing layer in a planar heterojunction thin-film architecture [28]. In early 2014, the efficiency was further increased to $16.7 \%$ using spiro-OMeTAD derivatives as hole-transporting materials [29]. Doping the $\mathrm{TiO}_{2}$ electron transport channel layer to enhance the charge carrier concentration, and modifying the ITO electrode to reduce its work function, allowed higher efficiencies up to $17.9 \%$ [30]. By 2015, an efficiency reaching 20.2\% was realized via 
deposition of high-quality $\mathrm{FAPbI}_{3}$ films, involving $\mathrm{FAPbI}_{3}$ crystallization by direct intramolecular exchange of dimethylsulfoxide (DMSO) molecules intercalated in $\mathrm{PbI}_{2}$ with formamidinium iodide [31]. In 2017, the introduction of additional iodide ions into the organic cation solution was reported, to form perovskite layers through a process of intramolecular exchange which decreases the concentration of deep-level defects; this allowed a certified power conversion efficiency of $22.1 \%$ [32]. Furthermore, by utilizing a fluorene-terminated hole-transport material with a finely-tuned energy level [33-40], Jeon et al. [33] fabricated high-efficiency perovskite solar cells with a state-of-the-art maximum efficiency of $23.2 \%$ (under reverse scanning) and a steady-state efficiency of $22.9 \%$ for small-area devices $\left(\sim 0.094 \mathrm{~cm}^{2}\right)$ and $21.7 \%$ for large-area devices $\left(\sim 1 \mathrm{~cm}^{2}\right)$. Eventually, certified efficiencies of $22.6 \%$ (small-area cells, $\sim 0.094 \mathrm{~cm}^{2}$ ) and $20.9 \%$ (large-area cells, $\sim 1 \mathrm{~cm}^{2}$ ) have been established [33]. Thus far, one of the record efficiency of $23.3 \%$ was held by the National Renewable Energy Laboratory (NREL) [5], as shown in Figure 1 Perovskite solar cells can achieve widely tunable bandgap energies. One of the strategically tunable bandgap optimization methods is to modify the bond distance and/or angle of $\mathrm{X}-\mathrm{Pb}-\mathrm{X}$ where $\mathrm{X}$ is a halide element [23, 25, 29]. Extensive efforts are dedicated to enhancing the optical absorption by the modification of a bandgap of the perovskite $\mathrm{MAPbI}_{3}$ layer.

Researchers have studied the impact of parametric conditions in the perovskite $\mathrm{MAPbI}_{3}$ film deposition and observed very interesting phenomena [27]. For example, the precursor temperature preceding the deposition can alter the bandgap for perovskite solar cells; they have found in this study that a film with a precursor temperature of $70^{\circ} \mathrm{C}$ shows an optimal band gap value $(1.516 \mathrm{eV})$ for solar cell applications. Moreover, when various processes are tuning by design, the reflectance data have demonstrated that varied fabrication techniques change the bandgaps [28] and that is studied in terms of the dielectric functions, optical bandgap, and optical absorption of the perovskite $\mathrm{MAPbI}_{3}$ films. The above variation has included such factors, for instance, differed spinning, evaporation, and various-solvent bathing. According to researchers, they have employed different deposition methods and processing techniques in the experimental design; the bandgap is studied to vary from $1.591 \mathrm{eV}$ to $1.501 \mathrm{eV}$ that is attributed to mechanisms such as different $\mathrm{Pb}-\mathrm{I}$ orbital hybridization and spin-orbit coupling.

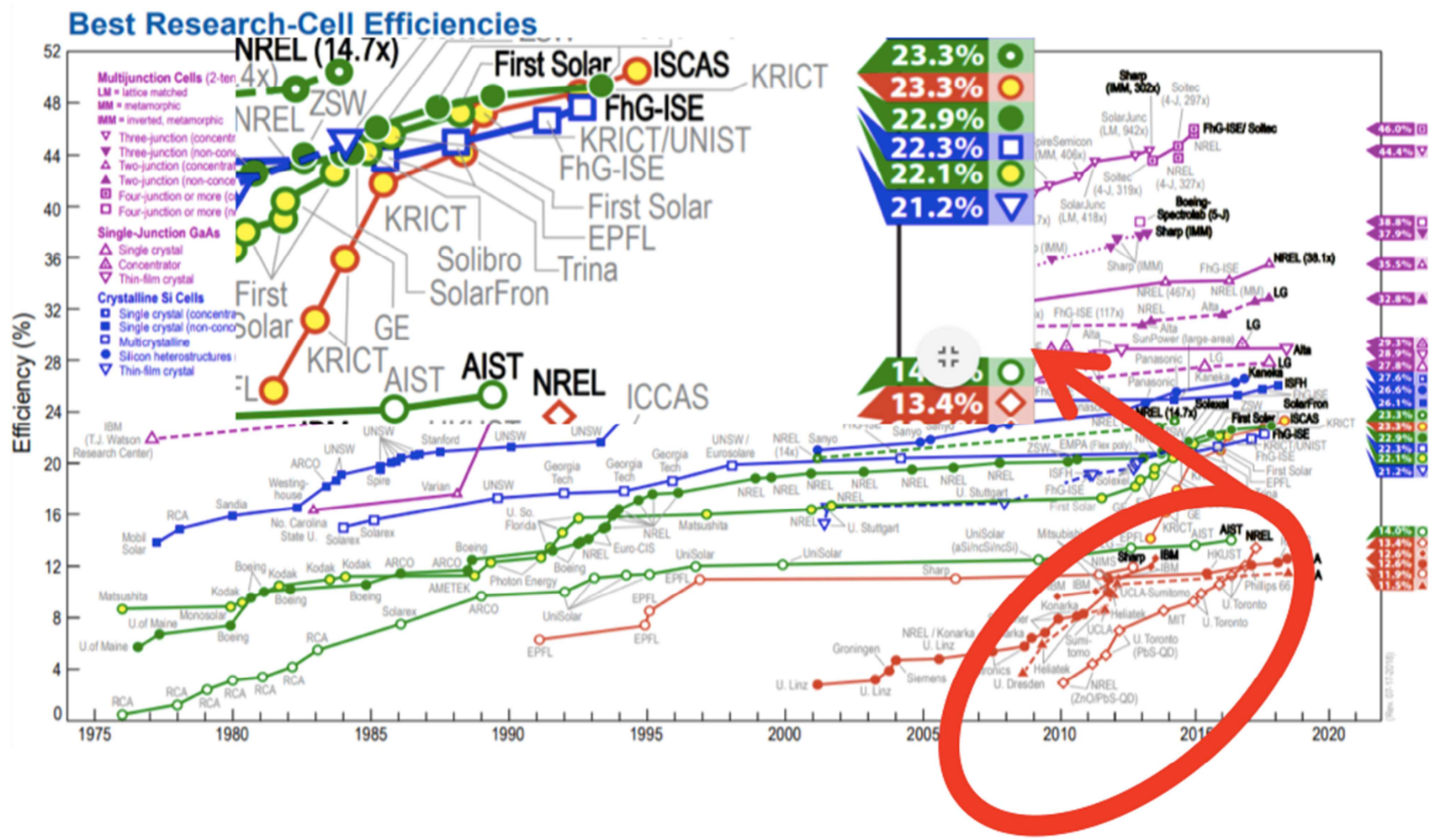

Figure 1. Best research cell efficiencies from the National Renewable Energy Laboratory (NREL) database [5] a plot of compiled values of highest confirmed conversion efficiencies for research cells, from 1976 to the present, for a range of photovoltaic technologies. Authors acknowledge the courtesy permit from NREL.

\subsection{Large Area Perovskite Solar Cells}

Another direction in perovskite research is to develop technologies to fabricate high-efficiency solar cells with large areas. Kim et al [3]. achieved a large-area $\left(16 \mathrm{~cm}^{2}\right)$ single perovskite solar cell with an independently certified efficiency of $12.1 \%$. An antisolvent spraying step realized a homogeneous and densely-packed perovskite film over a large area, and a metal grid was incorporated to remove the series resistance in the transparent conductor. Han et al [41]. 
employed a triple layer mesoporous $\mathrm{TiO}_{2} / \mathrm{ZrO}_{2} /$ carbon as a scaffold on a halide perovskite $(5-\mathrm{AVA})_{\mathrm{x}}(\mathrm{MA})_{1-\mathrm{x}} \mathrm{PbI}_{3}$, which allowed a $10.4 \%$-efficiency on an active area of $49 \mathrm{~cm}^{2}$, within a module consisting of 10 serially connected cells $\left(10 \times 10 \mathrm{~cm}^{2}\right)$. Moreover, a soft-cover deposition (SCD) method was used to successfully fabricate a perovskite solar cell with a total device area of $51 \mathrm{~cm}^{2}$; continuous processing in ambient air allowed a material utilization ratio up to $\sim 80 \%$, and a $17.6 \%$-efficiency was verified using a $1-\mathrm{cm}^{2}$ aperture by Han [42], Stolterfoht et al [43] applied PFN-P2 and $\mathrm{LiF}$ as interfacial layers at the PTAA/perovskite and perovskite/C60 interfaces, respectively, to suppress recombination losses by $65 \mathrm{meV}$ and $35 \mathrm{meV}$, respectively; hence the best efficiency of $20 \%$, and a certified efficiency of $19.83 \%$ for $1-\mathrm{cm}^{2}$ cells is achieved. Lu et al [44] modified the perovskite with cyano-substituted benzenethiol to enhance charge extraction and reduce charge recombination, thus increasing the efficiency from $19.0 \%$ (18.5\%) to $20.2 \%$ $(19.6 \%)$ with a $0.16-\mathrm{cm}^{2}\left(1.00-\mathrm{cm}^{2}\right)$ aperture.

\subsection{Stability Enhancement of Perovskite Solar Cells}

Any stability issues in perovskite solar cells arise from the material properties on which they are based, which are affected greatly by humidity, temperature, and light. Their thermal and environmental stability can be significantly enhanced when mixed-cations are used in perovskite solar cells. Saliba et al [44] introduced rubidium cations into perovskite solar cells to improve their reliability and photovoltaic performance; a $21.6 \%$-efficiency was achieved in a $0.5-\mathrm{cm}^{2}$ cell area, and $95 \%$ of this efficiency was retained under exposure to standard light for 500 hours. Certified efficiencies reached $20.1 \%$ and $19.5 \%$ for 0.049 $\mathrm{cm}^{2}$ and $1-\mathrm{cm}^{2}$ cell sizes, respectively, after Tan et. Al [45] reduced the interface recombination and contact resistance via excellent passivation achieved on chlorine-coated titanium oxide colloidal nanocrystals. The solar cell also demonstrated good stability, with $90 \%$ of the initial efficiency retained under light irradiation for $500 \mathrm{~h}$. Lee et al [46] applied a customized thin-film encapsulation comprising a multilayer stack of organic/inorganic layers deposited using chemical vapor deposition and atomic layer deposition; 97\% of its original efficiency was retained under accelerated conditions of $50 \mathrm{C}$ and $50 \%$ relative humidity for $300 \mathrm{~h}$. [8] Saidaminov et al [47] incorporated cadmium into a mixed perovskite lattice, thus releasing the remaining lattice strain, and further increasing the energetic penalty associated with vacancy formation (Figure 2A). Significantly extended stability is exhibited in unencapsulated perovskite solar cells, maintaining $>90 \%$ of their initial efficiency after 30 days of storage in ambient air at a relative humidity of $50 \%$ (Figure 2B). Better improvement has been attained on the life performance since then [14].

Authors should remark that the inorganic perovskite PSC has had rapid advancement in a few years and has played an extremely interesting role [48-54]. Solar cell efficiencies of devices using these materials have increased from $3.8 \%$ in 2009 to $25.2 \%$ in 2019 in single-junction architectures, and in silicon-based tandem cells, to $30 \%$ [55]. Several organicinorganic-based perovskite materials have recently been developed with standard wet chemistry techniques which is done in a traditional wet lab. The hybrid perovskite, i.e., methylammonium and formamidinium lead trihalides, has been fabricated employing solvent techniques, such as spin coating, slot-die coating, blade coating, spray coating, inkjet printing, and screen printing, electrodeposition, and vapor deposition techniques. The afore-mentioned solvent techniques have the potential to be scaled up with relative good feasibility. Interested readers are referred to recent literatures on the interesting inorganic and Pb-free PSC [4954]. The author's focus has been investigating a system via a typical systematic configuration and limiting this article with references to many excellent literatures for details.

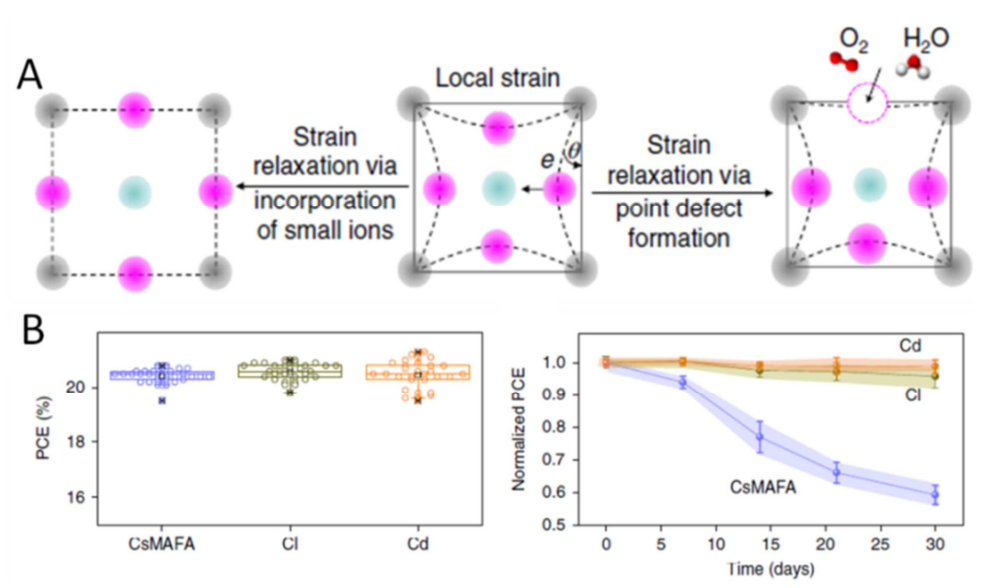

Figure 2. (A) Mechanisms of lattice relaxation. (B) Power conversion efficiency performance of perovskite solar cell with and without dopants. Figure revised from the literature [48]. Authors acknowledge a permit from its Copyright Clearance Center.

\subsection{Tandem Perovskite Silicon Solar Cell}

The best efficiency of the single-junction perovskite solar cell is as high as $23.3 \%$, but less than that of crystalline silicon $(26.67 \%)$, meaning that it is difficult for the former to compete effectively with silicon-based solar cells in the 
mainstream PV market. Perovskite/silicon tandem solar cells have very high efficiencies as they are an attractive route to break the Shockley-Queisser limit of single-junction solar cells. In fact, Bailie group from Stanford University reported in 2015 [56] the use of a simple "mechanical stacking" approach to obtaining a dual-junction tandem perovskite/silicon solar cell. By stacking a perovskite top cell of $12.7 \%$-efficiency atop a low-quality crystalline bottom cell with an efficiency of only $11.4 \%$, the solar cell conversion efficiency can be increased to $17 \%$. The following year, Bush et al [57]. successfully fabricated a four-terminal perovskite/silicon solar cell with an inversion structured top cell, yielding an efficiency of 18\%. Albrecht et al [58] reported an integrated perovskite/silicon heterojunction tandem cell by cryogenic treatment, allowing a maximum efficiency of $18.1 \%$. Furthermore, Chen et al [59]. reported stacking a record-high $16.5-\%$ efficiency semi-transparent perovskite top cell incorporating ultrathin metal electrodes, atop a $6.5-\%$ efficiency silicon heterojunction bottom cell, yielding an overall efficiency of $23.0 \%$. Werner et al [60] demonstrated that when a NIR transparent perovskite top cell (16.4\% efficiency) was mechanically stacked on a silicon sub-cell, the four-terminal and two-terminal efficiencies reached $25.2 \%$ and $20.6 \%$, respectively.

The successful development of perovskite/silicon tandem solar cells in Hong Kong Polytechnic University [61] yielded an efficiency as high as $25.5 \%$. This tandem cell was fabricated via three innovative approaches: 1) a chemical process/low-temperature annealing process in dry oxygen to reduce defect-related losses in the perovskite; 2) a tri-layer of molybdenum trioxide/gold/molybdenum trioxide with optimized constituent layer thicknesses to allow light penetration from the perovskite top layer into the silicon bottom layer; 3) a haze film that mimics the surface morphology of rose petals was applied as the top layer to trap more light. Werner et al [62] successfully fabricated a monolithic perovskite/silicon solar cell using a lowtemperature processing method after thickness optimization of the perovskite layer, producing efficiencies of $19.2 \%$ (for $1.22 \mathrm{~cm}^{2}$ ) and $21.2 \%$ (for $0.17 \mathrm{~cm}^{2}$ ). Bush et al [10]. increased the efficiency of monolithic, two-terminal, $1-\mathrm{cm}^{2}$ perovskite/silicon tandems to $23.6 \%$ by combining an infrared-tuned silicon heterojunction bottom cell with the recently developed caesium formamidinium lead halide perovskite. Furthermore, Sahli et al [63] achieved conformal growth of multiple compounds with controlled optoelectronic properties directly on micron-sized pyramids of textured monocrystalline silicon. Tandem devices have yielded a certified steady-state efficiency, $27.3 \%$ [20] by Osborne's group. These successive layers are combined in a series in the tandem perovskite to stop some substrate structure. In this series, as the solar radiation light incident upon the first perovskite layer, layer No. 1; the light energy out of the layer No. 2 is the light into the layer No. 2. The efficiency of the tandem device structure as a combined system, is the total energy output in series divided by the energy supplied to the first layer. Theoretically, a tandem structure that connects in series of n-layers $[i=1,2 \ldots n]$ can achieve the following system efficiency:

$$
1-\eta_{\text {series }}=\prod_{i}\left(1-\eta_{i}\right)
$$

\subsection{Energy Storage}

When it is connected to the grid, the variable power output from a large amount of renewable energy (e.g. photovoltaic or wind energy) may lead to huge power fluctuations. The fluctuations can range from a few seconds to tens of minutes, thus affecting the grid frequency and causing grid instability. As a result, power demand leveling by energy storage becomes increasingly important.

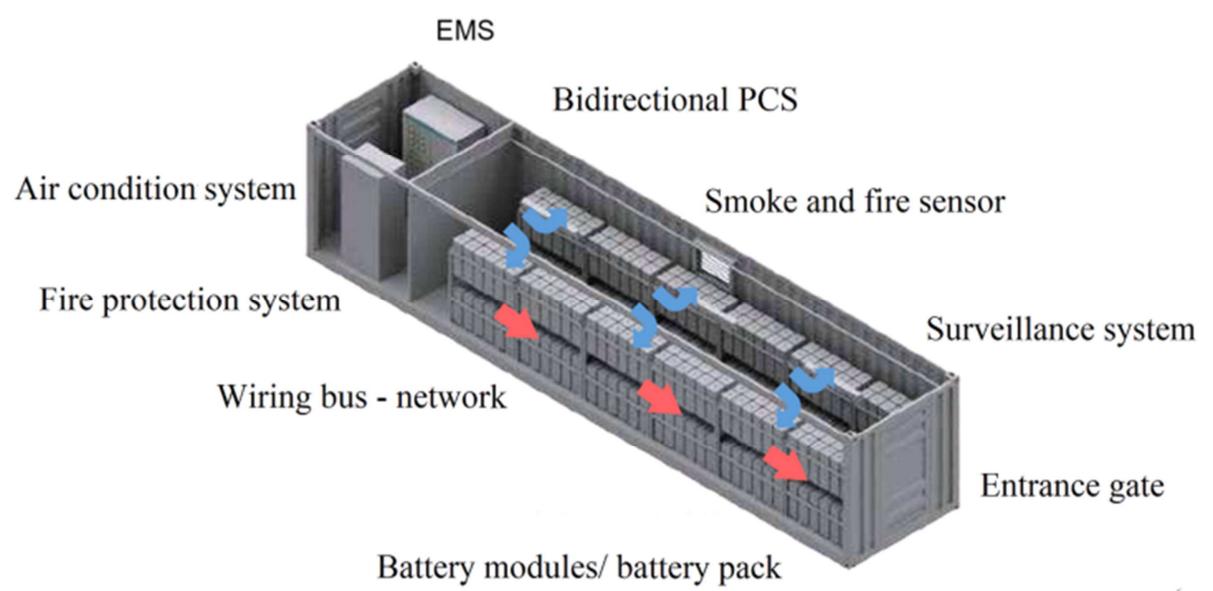

Figure 3. Illustrated is the interior of an energy storage. Its energy management system is located atop for the operator's interface.

Researchers have successfully constructed storage banks cargo by Liu, et al [16], and a bank of the energy storage systems by Kittner, et al [4]. The energy storage is a cargo container with battery in banks stacking racks as shown in
Figure 3. They are pretty innovative and have earned a noteworthy top innovation-2017 awarded in its field [16]. This construction is a solution from the supply-side strategies with storage capability of $3 \mathrm{MWh}$ that is contained in a cargo 
of battery stacking racks for the energy storage. Both the current energy storage design and system development is based on lithium-ion batteries, car batteries or lead-acid batteries; there is a huge opportunity for new and great innovation in the storage technology area to date. A storage cargo container typically includes the battery bank, the stacking rack (s), electrical wiring/connection/ harness for both global and local wiring, sensors and video monitors, air conditioning, and fire safety system. The energy storage system is connected to the national grid and has been tested for an extended period of time.

The method, that resolves the imbalance of the need and supply between a consumer who is in need of power and a prosumer in supply, abides by a simple principle as follows. The surplus energy production in day time is stored in the energy storage devices; and this energy is extracted for use when it is needed later or deemed convenient.

Furthermore, the energy storage system has a designed-in battery management system to ensure that every battery cells are integrated optimally and are operating in the safe operation window via both charge and discharge processes.

A good energy management system is critical so that it has significantly improved the battery efficiency and the battery capacity. The illustration in Figure 3 is a design of an integrated energy storage system. It has operated in $3 \mathrm{MWh}$ capacity [16]. A typical energy storage system includes the following: battery modules, wiring bus, fire protection system, air conditioning system, bidirectional power conversion via smart grid, smoke and fire sensor, surveillance system, and entrance for the engineering works.

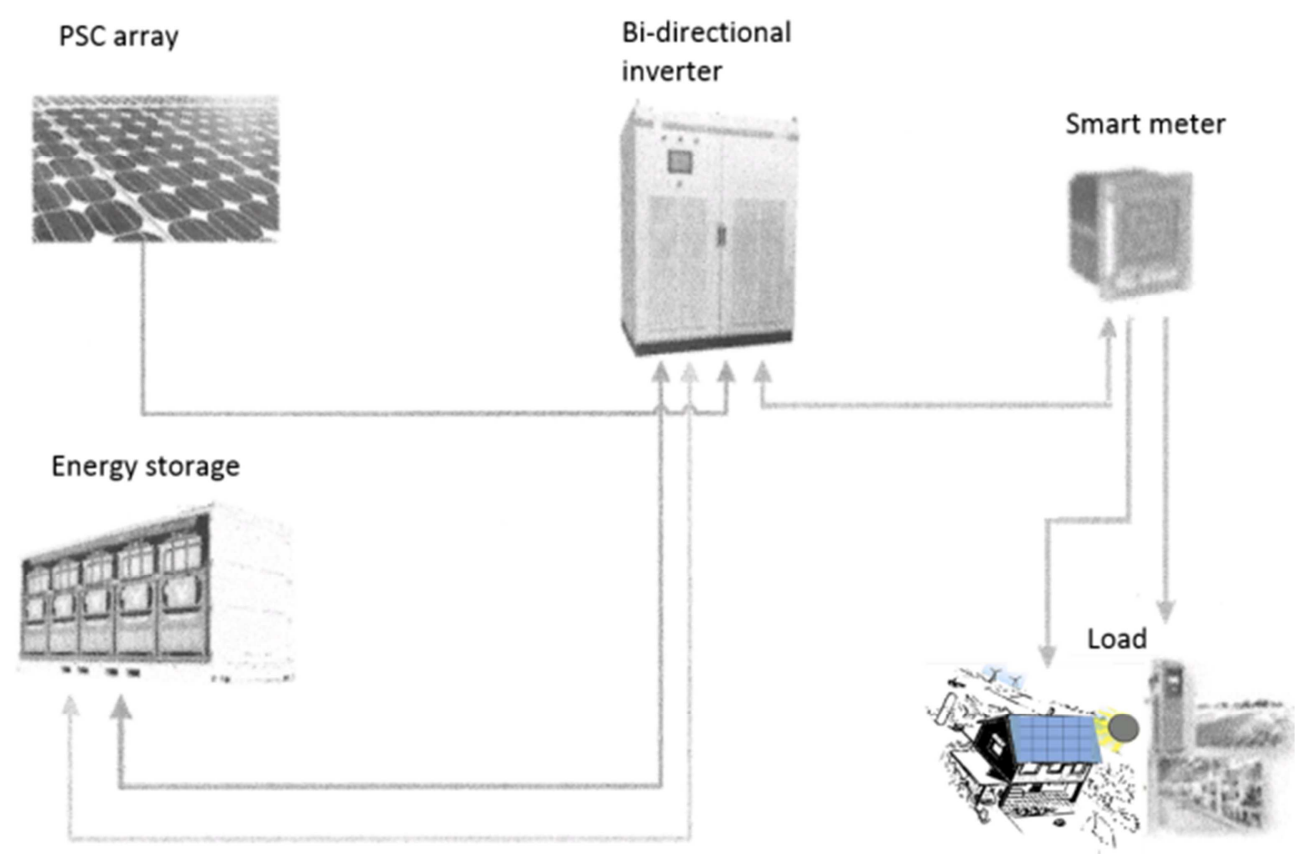

Figure 4. The design schematics illustrates a large-scale solar power generation system.

Figure 4 shows a design schematics of an integrated solar PV power generation system that is designed and built for the local power generation. The utility companies often offer a gateway for the local power generation to be connected to a power grid through a smart meter that can transfer electric energy either to or from the power grid; this power transfer can meet the requirement the peak and valley hours' use of electricity. The gateway is connected to a smart meter; the smart meter can have the necessary energy exchange that is available in connection to the power grid and that provides power to a consumer load.

Finally, a strategy is employed to smooth the peak power both from the supply-side and in the demand-side of the power. This strategy is imperative for the power dispatch; this strategy can help the good transmission in order to resolve field issues during the power dispatch uptime. The improvement in the uptime will be beneficial for the clean energy from resources like the perovskite solar energy power. The energy efficiency needs to be stable by balancing supply and demand. With the improved design of energy storage systems, the transmitted values of the outputs from renewable energy sources are believed to improve significantly and to become more stable on a large time scale.

\section{Discussion}

The perovskite solar cell presents itself a very promising opportunity for the solar clean energy technology. Along with other emerging clean technologies, it exists side-by-side with a young PV industry that is highly successful and yet fragmented to date in the energy market in comparison to the traditional fossil-based energy incumbent. Both industries have great technology profiles and market potentials for the energy market. In keeping pace to become mature, they need huge growth into the traditional one. Because of their potentials, they are ranked as highly attractive as its profiled success will need more competitive advantage for both investors and consumers. 
The development of perovskite solar cells has advanced favorably for their entrance into many mainstream applications. The potential of perovskite can realize far higher efficiencies, environmental and performance stability at minimal production costs. This potential will promote perovskites to be commercially mature with the benefits of high efficiency, easy to manufacture, and cost advantages..

Authors have shown that the perovskite solar cell technology has achieved very high energy conversion efficiency. Simply put, this technology could become a huge contender in the PV industry with tremendous potential and low cost to produce if these cells could be functionally produced with a long-term stability. They demonstrated that these cells are prepared well in various topologies in the cell structure helpful for the performance improvement including but not limited to the tandem designs, encapsulation in multilayer structure, planar architecture with new materials of varied bandgap, etc.

The tandem designs, such as perovskite cells stacked atop silicon sub-cells have achieved over $27 \%$ in efficiency and above. They can have very high efficiencies and has recently attracted a huge and dramatically increasing attention from researchers.

Perovskite solar cells have advantages for widely tunable energy bandgap via variation of the dopant concentration. The single-junction efficiencies of these perovskite solar cells are comparable to those of multi-crystalline silicon, cadmium telluride and copper indium gallium selenide. As the research and development has exhibited significant success in the energy storage technology at a mega-watt level, authors have briefly reviewed the energy storage [4, 16] from the supply side management. This is important for the expanding technologies in successful alternative energy generation and application.

\section{Conclusions}

The scope of this article is to study an emerging solar energy system highlighted by both advances of the perovskite solar cells and of energy storages. In conclusion, authors have provided case studies on solar technologies that there are tremendous research and important breakthrough in perovskite solar cells development. In the afore-mentioned cases, the related new technologies have potentially superior performance with promising new materials, new processing methods, and superior architecture. The perovskite solar cells have high efficiency at near or above $30 \%[20,55]$, and the PSC have extended life-time at or above one-year.

In the light of alternative energy technologies, authors emphasize PSC and they briefly discuss the significant milestone progress in the energy storage technology. As the exciting research and development has exhibited significant success in energy storage technology at a meg-watt hours level, a study on a distributed clean energy system is conducted.
Finally, the huge commitment and intense international collaboration among scientists have been dedicated to the perovskite stability [8, 10-12] and have extended beyond its one-year long life-time in just a few years of development. The perovskite is apparently providing the fastest-advancing solar technology to date; authors therefore believe that may be available soon for emerging technology commercialization in scale as this technology matures in near future.

\section{Declaration}

Ethics approval and consent to participate (Yes)

Consent for publication (Yes)

Availability of data and material (Yes)

Competing interests (Not applicable)

Funding (Yes)

Authors' contributions (Yes, cf. below)

Conceived plan: aj, kc, jz. Investigation: zl, zy, kc, aj. Data analysis: zl, aj, zy, kc, jz, jz. Wrote the paper: aj, zl, zy, kc.

Authors have no competing financial interests.

Acknowledgements (Yes)

Authors' information (optional) (Yes, cf. below)

\section{Abbreviation}

DMSO: dimethylsulfoxide

ES: Energy Storage

ITO: indium tin oxide

MW: mega watt

MWh: mega watt hour

NREL: National Renewable Energy Laboratory

PURPA: public utility regulatory policies act

PV: photovoltaics

\section{Acknowledgements}

Financial support is acknowledged, from the National Natural Science Foundation of China (grant number 61650110517).

\section{References}

[1] Woodrow W, Clark (2010). Sustainable Communities Design Handbook". Germany, Elsevier Press. 65-81.

[2] Woodrow W, Clark. AJ, Jin (2018). Sustainable Cities and Communities Design Handbook. Green Engineering, Architecture, and Technology. 111-128.

[3] The Public Utility Regulatory Policies Act. Public Utility Regulatory Policies Act is a United States Act passed as part of the National Energy Act. https://en.wikipedia.org/wiki/Public_Utility_Regulatory_Polic ies_Act.

[4] Kittner N, Lill F, Kammen D M (2017). Energy storage deployment and innovation for the clean energy transition. Nature Energy. 2 (9): 17125. 
[5] NRL, a Collection of National Renewable Energy Laboratory database enlists the best research cells efficiency confirmed for research cells, from 1976 to the present, for a range of photovoltaic technologies; https://www.nrel.gov/pv/assets/pdfs/pv-efficiencies-07-172018.pdf.

[6] Park N G, Grätzel M, Miyasaka T, et al (2016). Towards stable and commercially available perovskite solar cells. Nature Energy. 1 (11): 16152.

[7] Correa-Baena J P, Abate A, Saliba M, et al (2017). The rapid evolution of highly efficient perovskite solar cells. Energy Environ. Sci. 10 (3): 710-727.

[8] Christians J A, Schulz P, Tinkham J S, et al (2017). Tailored interfaces of unencapsulated perovskite solar cells for $>1,000$ hour operational stability. 3 (1): 68-74.

[9] KhalidMahmood, ArshiKhalid, \& Taqimehran, M. (2019). Mapbi 3 microneedle-arrays for perovskite photovoltaic application. Nanoscale Advances, 1.

[10] Bush K A, Palmstrom A F, Yu Z J, et al (2017). 23.6\%efficient monolithic perovskite/silicon tandem solar cells with improved stability. Nature Energy. 2 (4): 17009.

[11] Wu W Q, Wang Q, Fang Y, et al (2018). Molecular doping enabled scalable blading of efficient hole-transport-layer-free perovskite solar cells. Nature Communications. 9 (1): 1625.

[12] Zhang X, Ren X, Liu B, et al (2017). Stable High Efficiency Two-Dimensional Perovskite Solar Cells via Cesium Doping. Energy Environ. Sci. 10 (10): 2095-2102.

[13] Eperon G E, Leijtens T, Bush K A, et al (2016). Perovskiteperovskite tandem photovoltaics with optimized bandgaps. Science. 354 (6314). 861-865.

[14] Grancini G, Roldán-Carmona, C, Zimmermann I, et al (2017). One-Year stable perovskite solar cells by 2D/3D interface engineering. Nature Communications. 8: 15684.

[15] US 4668595, Yoshino; Akira, "Secondary Battery", issued 10 May 1985, assigned to Asahi Kasei.

[16] M. Liu and Y. Xu; "Energy Storage Applications 2017" Top10 award; The 1st International Conference on Energy Storage Materials (ICENSM 2017).

[17] Urbain F, Murcia-López S, Nembhard N, et al (2019). Solar vanadium redoxflow battery powered by thin-film silicon photovoltaics for efficient photoelectrochemical energy storage. Phys D-Appl Phys, 52: 044001.

[18] Lei B, Li G R, Chen P, et al (2017). A solar rechargeable battery based on hydrogen storage mechanism in dual-phase electrolyte. Nano Energy. 38: 257-262.

[19] Liao W M, Zhang J H, Hou Y J, et al (2016). Visible-lightdriven $\mathrm{CO} 2$ photocatalytic reduction of $\mathrm{Ru}$ (II) and Ir (III) coordination complexes. InOrg Chem Commun. 73: 80-89.

[20] Mark Osborne. Oxford PV takes record perovskite tandem solar cell to $27.3 \%$ conversion efficiency. https://www.pvtech.org/news/oxford-pv-takes-record-perovskite-tandemsolar-cell-to-27.3-conversion-effi

[21] Juarez-Perez, Emilio J, Ono Luis K, Maeda, et al (2018). Photodecomposition and thermal decomposition in methylammonium halide lead perovskites and inferred design principles to increase photovoltaic device stability. Journal of
Materials Chemistry A. 6 (20). 9604-9621.

[22] Chynoweth, A. G (1960). Pyroelectricity, Internal Domains, and Interface Charges in Triglycine Sulfate. Physical Review. 117 (5): 1235-1243.

[23] Chen, F. S (1969). Optically Induced Change of Refractive Indices in $\mathrm{LiNbO} 3$ and $\mathrm{LiTaO} 3$. Journal of Applied Physics. 40 (8): 3389.

[24] Kim, H.-S., Lee, C.-R., Im, J.-H., Lee, K.-B., Moehl, T., Marchioro, A., Park, N.-G (2012). Lead Iodide Perovskite Sensitized All-Solid-State Submicron Thin Film Mesoscopic Solar Cell with Efficiency Exceeding 9\%. Scientific Reports. 2 (1).

[25] Lee M M, Teuscher J, Miyasaka T, et al (2012). Efficient Hybrid Solar Cells Based on Meso-Superstructured Organometal Halide Perovskites. Science, 338 (6107): 643-647.

[26] Ball J M, Lee M M, Hey A, et al (2013). Low-temperature processed meso-superstructured to thin-film perovskite solar cells. Energy \& Environmental Science. 6 (6): 1739-1743.

[27] Burschka J, Pellet N, Moon S J, et al (2013). Sequential deposition as a route to high-performance perovskitesensitized solar cells. Nature. 499 (7458): 316-319.

[28] Liu M, Johnston M B, Snaith H J (2013). Efficient planar heterojunction perovskite solar cells by vapour deposition. Nature. 501 (7467): 395.

[29] Jeon, N. J., Lee, H. G., Kim, Y. C., Seo, J., Noh, J. H., Lee, J., \& Seok, S (2014). io-Methoxy Substituents in SpiroOMeTAD for Efficient Inorganic-Organic Hybrid Perovskite Solar Cells. Journal of the American Chemical Society. 136 (22): 7837-7840.

[30] J. Cao, B. Wu, R. Chen, Y. Wu, Y. Hui, B. W. Mao, and N. Zheng (2018); A Multi - Yolk-Shell Structured Nanocatalyst Containing Sub - $10 \mathrm{~nm}$ Pd Nanoparticles in Porous CeO2. Advanced materials. 30 (11), 1705596.

[31] Yang W S, Noh J H, Jeon N J, et al (2015). High-performance photovoltaic perovskite layers fabricated through intramolecular exchange. Science. 348 (6240): 1234.

[32] Yang W S, Park B W, Jung E H, et al (2017). Iodide management in formamidinium-lead-halide-based perovskite layers for efficient solar cells. Science. 356 (6345): 1376-1379.

[33] Jeon N J, Na H, Jung E H, et al (2018). A fluorene-terminated hole-transporting material for highly efficient and stable perovskite solar cells. Nature Energy, 3 (8).

[34] Park, Nam-Gyu (2015). Perovskite solar cells: an emerging photovoltaic technology. Materials Today, 18 (2): 65-72.

[35] Löper P, Stuckelberger M, Niesen B, et al (2015). Complex Refractive Index Spectra of $\mathrm{CH}_{3} \mathrm{NH}_{3} \mathrm{PbI}_{3}$ Perovskite Thin Films Determined by Spectroscopic Ellipsometry and Spectrophotometry. Journal of Physical Chemistry Letters. 6 (1): 66-71.

[36] Chen W, Wu Y, Yue Y, et al (2015). Efficient and stable large-area perovskite solar cells with inorganic charge extraction layers. Science. 350 (6263): 944-948.

[37] Zhu X, Su H, Marcus R A, et al (2014). Computed and Experimental Absorption Spectra of the Perovskite $\mathrm{CH}_{3} \mathrm{NH}_{3} \mathrm{PbI}_{3}$. Journal of Physical Chemistry Letters. 5 (17): 3061 . 
[38] Li W, Zhao K, Zhou H, et al (2018). Precursor solution temperature dependence of optical constants, band gap and Urbach tail in organic-inorganic hybrid halide Perovskite films. Journal of Physics D Applied Physics. 52 (4): 045103

[39] Li W, Sha T, Wang Y, et al (2017). Effects of deposition methods and processing techniques on band gap, interband electronic transitions, and optical absorption in perovskite $\mathrm{CH}_{3} \mathrm{NH}_{3} \mathrm{PbI}_{3}$ films. Applied Physics Letters. 111 (1), 011906.

[40] Kim J, Yun J S, Cho Y, et al (2017). Overcoming the Challenges of Large-Area High-Efficiency Perovskite Solar Cells. Acs Energy Letters. 2 (9): 1978-1984.

[41] Ye F Tang W, Xie F, et al (2017). Low-Temperature SoftCover Deposition of Uniform Large-Scale Perovskite Films for High-Performance Solar Cells. Advanced Materials. 29 (35): 1701440.

[42] Stolterfoht M, Wolff C M, Márquez J A, et al (2018). Visualization and suppression of interfacial recombination for high-efficiency large-area pin perovskite solar cells. Nature Energy. 3 (10).

[43] Lu J, Lin X, Jiao X, et al (2018). Interfacial benzenethiol modification facilitates charge transfer and improves stability of $\mathrm{cm}$-sized metal halide perovskite solar cells with up to $20 \%$ efficiency. Energy \& Environmental Science. 11 (7): 10.1039.

[44] Saliba M, Matsui T, Domanski K, et al (2016). Incorporation of rubidium cations into perovskite solar cells improves photovoltaic performance. Science. 354 (6309): 206.

[45] Tan H, Jain A, Voznyy O, et al (2017). Efficient and stable solution-processed planar perovskite solar cells via contact passivation. Science. 355 (6326): 722-726.

[46] Lee Y I, Jeon N J, Kim B J, et al (2017). A Low-Temperature Thin-Film Encapsulation for Enhanced Stability of a Highly Efficient Perovskite Solar Cell. Advanced Energy Materials. 8 (9): 1701928

[47] Saidaminov M I, Kim J, Jain A, et al (2018). Suppression of atomic vacancies via incorporation of isovalent small ions to increase the stability of halide perovskite solar cells in ambient air. Nature Energy. 3 (8): 648-654.

[48] Snaith (2013), Henry J. Perovskites: The Emergence of a New Era for Low-Cost, High-Efficiency Solar Cells. The Journal of Physical Chemistry Letters. 4 (21): 3623-3630.

[49] Noel, N. K., Stranks, S. D., Abate, A., Wehrenfennig, C., Guarnera, S., Haghighirad, A.-A., ... Snaith, H. J (2014). Leadfree organic-inorganic tin halide perovskites for photovoltaic applications. Energy Environ. Sci. 7 (9), 3061-3068.

[50] Abate, Antonio; Saliba, Michael; Hollman, Derek J.; Stranks, Samuel D.; Wojciechowski, Konrad; Avolio, Roberto; Grancini, Giulia; Petrozza, Annamaria; Snaith, Henry J (2014). Supramolecular Halogen Bond Passivation of OrganicInorganic Halide Perovskite Solar Cells. Nano Letters. 14 (6): $3247-3254$
[51] Tai, Q., Tang, K.-C., \& Yan, F (2019). Recent progress of inorganic perovskite solar cells. Energy \& Environmental Science. 12, 2375-2405

[52] Khan, U., Zhinong, Y., Khan, A. A, Zulfiqar, A., \& khan, Q. U (2019). Organic-inorganic hybrid perovskites based on methylamine lead halide solar cell. Solar Energy. 189, 421425.

[53] Li, Z., Klein, T. R., Kim, D. H., Yang, M., Berry, J. J., van Hest, M. F. A. M., \& Zhu, K (2018). Scalable fabrication of perovskite solar cells. Nature Reviews Materials. 3 (4), 18017.

[54] Chen, H., Xiang, S., Li, W., Liu, H., Zhu, L., \& Yang, S (2018). Inorganic Perovskite Solar Cells: A Rapidly Growing Fiel. Solar RRL. 2 (2), 1700188.

[55] Emiliano Bellini. Netherlands' ECN achieves 30.2\% efficiency for bifacial tandem cell based on perovskite. https://www.pv-magazine.com/2019/03/04/netherlands-ecnachieves-30-2-efficiency-for-bifacial-tandem-cell-based-onperovskite/

[56] Bailie C D, Christoforo M G, Mailoa J P, et al (2014). Semitransparent perovskite solar cells for tandems with silicon and CIGS. Energy \& Environmental Science, 8 (3): 956-963.

[57] Bush K A, Bailie C D, Chen Y, et al (2016). Thermal and Environmental Stability of Semi-Transparent Perovskite Solar Cells for Tandems Enabled by a Solution-Processed Nanoparticle Buffer Layer and Sputtered ITO Electrode. Advanced Materials. 28 (20): 3937-3943.

[58] Albrecht S., Saliba M., Correa Baena J. Lang P., F., Kegelmann L., Mews M., Steier L., Abate A., Rappich J., Korte L., Schlatmann R., Nazeeruddin M. K., Hagfeldt A., Grätzel M., Rech B (2015). Monolithic Perovskite/SiliconHeterojunction Tandem Solar Cells Processed at Low Temperature. Energy Environ. Sci. 9 (1): 81-88.

[59] Chen B, Bai Y, Yu Z, et al (2016). Efficient Semitransparent Perovskite Solar Cells for $23.0 \%$ - Efficiency Perovskite/ Silicon Four-Terminal Tandem Cells. Advanced Energy Materials. 6 (19): 1601128.

[60] Werner J, Barraud L, Walter A, et al (2016). Efficient NearInfrared-Transparent Perovskite Solar Cells Enabling Direct Comparison of 4-Terminal and Monolithic Perovskite/Silicon Tandem Cells. 1, 474-480.

[61] Charles Chee Surya,; PolyU Develops Perovskite-Silicon Tandem Solar Cells with the World's Highest Power ConversionEfficiency.https://www.polyu.edu.hk/web/en/medi a/media_releases/index_id_6208.html

[62] Werner J, Weng C H, Walter A, et al (2016). Efficient Monolithic Perovskite/Silicon Tandem Solar Cell With Cell Area $>1 \mathrm{~cm}$ (2). Journal of Physical Chemistry Letters. 7 (1): $161-166$.

[63] Sahli F, Werner J, Kamino B A, et al (2018). Fully textured monolithic perovskite/silicon tandem solar cells with $25.2 \%$ power conversion efficiency. Nature Materials. 17 (9). 